\title{
Author Correction: Evolution of charge order topology across a magnetic phase transition in cuprate superconductors
}

Mingu Kang (D), Jonathan Pelliciari, Alex Frano, Nicholas Breznay (D), Enrico Schierle, Eugen Weschke, Ronny Sutarto (D), Feizhou He (D), Padraic Shafer DD, Elke Arenholz, Mo Chen, Keto Zhang, Alejandro Ruiz, Zeyu Hao, Sylvia Lewin (D), James Analytis, Yoshiharu Krockenberger, Hideki Yamamoto (ID, Tanmoy Das and Riccardo Comin (D)

Correction to: Nature Physics https://doi.org/10.1038/s41567-018-0401-8, published online 21 January 2019.

The authors wish to add the following sentence in the Acknowledgements: “The authors are grateful to S. Kivelson, B. Fine, K. M. Shen and D. Hawthorn for insightful discussions." The online versions of this Letter have been amended accordingly.

Published online: 28 May 2019

https://doi.org/10.1038/s41567-019-0569-6 